\title{
Extracting Agent-Based Models of Human Transportation Patterns
}

\author{
Rahmatollah Beheshti \\ Department of EECS \\ University of Central Florida \\ Orlando, Florida 32816 \\ Email: beheshti@knights.ucf.edu
}

\author{
Gita Sukthankar \\ Department of EECS \\ University of Central Florida \\ Orlando, Florida 32816 \\ Email: gitars@eecs.ucf.edu
}

\begin{abstract}
Due to their cheap development costs and ease of deployment, surveys and questionnaires are useful tools for gathering information about the activity patterns of a large group and can serve as a valuable supplement to tracking studies done with mobile devices. However in raw form, general survey data is not necessarily useful for answering predictive questions about the behavior of a large social system. In this paper, we describe a method for generating agent activity profiles from survey data for an agent-based model (ABM) of transportation patterns of 47,000 students on a university campus. We compare the performance of our agent-based model against a Markov Chain Monte Carlo (MCMC) simulation based directly on the distributions fitted from the survey data. A comparison of our simulation results against an independently collected dataset reveals that our ABM can be used to accurately forecast parking behavior over the semester and is significantly more accurate than the MCMC estimator
\end{abstract}

\section{INTRODUCTION}

Agent-based simulations have been used successfully for modeling human social systems in diverse fields including economics, sociology, anthropology, and archaeology [1]. A perennial question that arises in the development of an agentbased simulation is how to initialize the models to create a realistic population of agents. In simple models with few parameters, it is feasible to perform a sensitivity analysis to explore the effects of the parameters on the performance of the simulation. However in more complicated agent decisionmaking models, creating a realistic population of agents can be challenging due to the larger range of parameters governing the behavior of the simulated entities.

Surveys and questionnaires can be used to collect an accurate static snapshot of the behavior of large social systems but lack the predictive power of simulations. It is more difficult to explore "what-if" questions with a survey since posing questions to participants about hypothetical scenarios can be problematic due to human cognitive biases such as anchoring or risk-aversion. In this paper, we show how both methodologies, surveying and agent-based simulation, can be combined to model human social systems with higher verisimilitude and to explore the ramifications of different behavior patterns and trends.

This paper specifically addresses the problem of creating individual agent profiles for an activity-based microsimulation model of transportation, dining, parking, and building occupation preferences on a large university campus. One problem with agent-based models is that linking the models and simulation processes with the observed data is challenging. The main contribution of our research is to demonstrate a procedure for systematically linking the observed survey data of people's transportation preferences with an executable agent model. In contrast, stochastic simulation approaches such as Markov Chain Monte Carlo (MCMC), have been used to forecast the outcome of temporal processes and are simple to create and initialize from observed data [2]. However, in our results, we show that our method is substantially more accurate at forecasting future effects than an MCMC estimator initialized from the same survey data, even at answering relatively simple questions. An additional benefit is that manipulating the operation of an agent-based model can empower researchers with better intuitions about the reasons behind emerging group phenomena rather than merely observing the unfolding of a stochastic process [3].

Urban simulation is a particularly fertile area for agentbased simulation research since it requires modeling a large number of interdependent agents making sequential decisions within a small region. Benenson et al. [4] present two motivations for defining urban agents, as a distinct group within the general class of autonomous agents:

1) urban agents often have a high degree of mobility resulting in rapidly changing spatial relationships.

2) to succeed, urban agents require a strong capability to perceive and adapt to the evolving urban environment shaped by neighboring agents.

In a general urban model, there can be many classes of agents-developer agents constructing new buildings, car agents moving in traffic, business agents providing services to customer agents, and land-use agents who own and manage parcels and lots [4]. In our model, we focus on modeling transient activity patterns such as transportation habits, dining preferences, and building occupation times. The goal is to predict the large-scale aggregate activity patterns of thousands of students over the duration of the semester, in contrast to work that has been done on learning individual transportation modality and route preferences using cell phone and GPS data from hundreds of individuals (e.g., the MIT Reality Mining 
project [5] or the Microsoft Multiperson Location survey [6]). An alternate approach, crowdsourcing, leverages the "wisdom of the crowd" to answer simple queries and has been demonstrated to be a useful tool for gathering specialized real-time data for various transportation related activities such as gas pricing (GasBuddy) or parking spot detection (OpenSpot). It can be a useful replacement for questionnaires and surveys in cases where some incentive exists for users to install software and self-report on their behavior. These technologies are highly complementary, and in this paper we demonstrate how the models from our activity-based microsimulation can supplement mobile device monitoring and crowdsourcing, enabling accurate transportation forecasting and exploration of hypothetical scenarios.

This paper is organized as follows. In the next section, we describe the process of extracting agent-based models from survey data and our activity-based microsimulation. Then we describe the construction of a benchmark Markov Chain Monte Carlo simulation in Section III. Section IV presents an evaluation of our proposed agent-based simulation initialization model on a simple parking forecasting problem. We conclude by describing other related work in simulating urban social systems and transportation forecasting.

\section{MethoD}

In this section, we describe the development process for our activity-based microsimulation, including the agent-based model, survey data collection, activity profile generation, path planning, and simulation system. For our urban region, we selected the University of Central Florida main campus, which is one the biggest academic institutions in the US with almost 59,000 students and 10,567 staff. It is adjacent to the Central Florida Research Park which is home to 116 companies with approximately 9,500 employees. The presence of nearby businesses and existence of commuters traveling between multiple UCF campuses give rise to a social system with a diverse and complex set of transportation patterns.

\section{A. Data Collection}

To simplify the data collection process, our initial study focused solely on modeling student transportation, dining, and building occupancy patterns. 1003 students responded to our online survey posted on KwikSurveys which was advertised on various campus email lists. The questions on the survey were grouped into six different categories, related to possible places that could be visited on the main campus:

1) Daily attendance patterns, including the days and times that the participant arrives and departs the main campus

2) Initial location, either the dorm (for on-campus students) or the entrance that was used to enter the campus (for commuting students)

3) Visitation frequency for on-campus dining locations

4) Usage patterns for recreation and athletic facilities

5) Usage of administrative and other miscellaneous locations

6) Frequency of parking lot and shuttle stop usage
For categories three through six, students were specifically queried about their visitation frequencies. For these questions, responses included one of: never, rarely, once a month, several times in a month, once a week, several times in a week and every day.

In addition to the survey data, our agent-based simulation used publicly available statistics about $\mathrm{UCF}^{*}$ and the main campus building map ${ }^{\dagger}$. A graph of the campus paths and roads was created from the main campus building map. The set of nodes in the graph is the union of the locations in the survey plus the junctions between the streets and pathways. The edges of this graph represent the roads and walkways among the nodes. The weights of the edges show the distance between the connecting nodes. Each node and edge has a tag. This tag for the nodes indicates whether they are a location of interest on the map or merely a junction. For example, a department is a location of interest, and a junction created by intersecting two roads or walkways is not. The tag for the edges determines whether they are walkways or roads. Figure 1 shows a snapshot of the map and also the corresponding graph in the background.

\section{B. Agent-Based Model}

To perform transportation forecasting on the UCF campus, we created an agent-based model for simulating the common activities (transportation, dining, recreation, and building occupancy) performed by the 47,000 students on the main campus. Each agent in the model represents an individual student and has a unique set of parameters that govern his/her activity profile. An agent's defining parameters are: entrance, dormitory, department, class building, arrive, depart, lunch, dinner, beverage, recreation and wellness, parking, shuttle, and miscellaneous. The first four parameters designate the single (most common) value of the agents' entry point to the campus, housing situation, home department, and main class building. Note that we did not explicitly represent the students' class schedules in the model. Even though this would have improved the fidelity of the model, we felt that addition would not generalize well to other types of urban models. Arrive and depart are lists showing the times the agent enters the campus and leaves it. The remaining parameters are lists of locations for the agent's dining, recreation, and commuting. Additionally, each parameter that includes a location has another matching parameter that shows the time or frequency of visiting that location.

In this paper, we explore two agent-based modeling methods:

- ABM only: agent model parameters are randomly sampled from a uniform distribution over a realistic range of values. This method is commonly used in a most of the ABM systems described in our related work overview and is used as a benchmark for our proposed method.

\footnotetext{
${ }^{*}$ http://www.iroffice.ucf.edu/character/current.htm

${ }^{\dagger}$ http://map.ucf.edu/printable/
} 


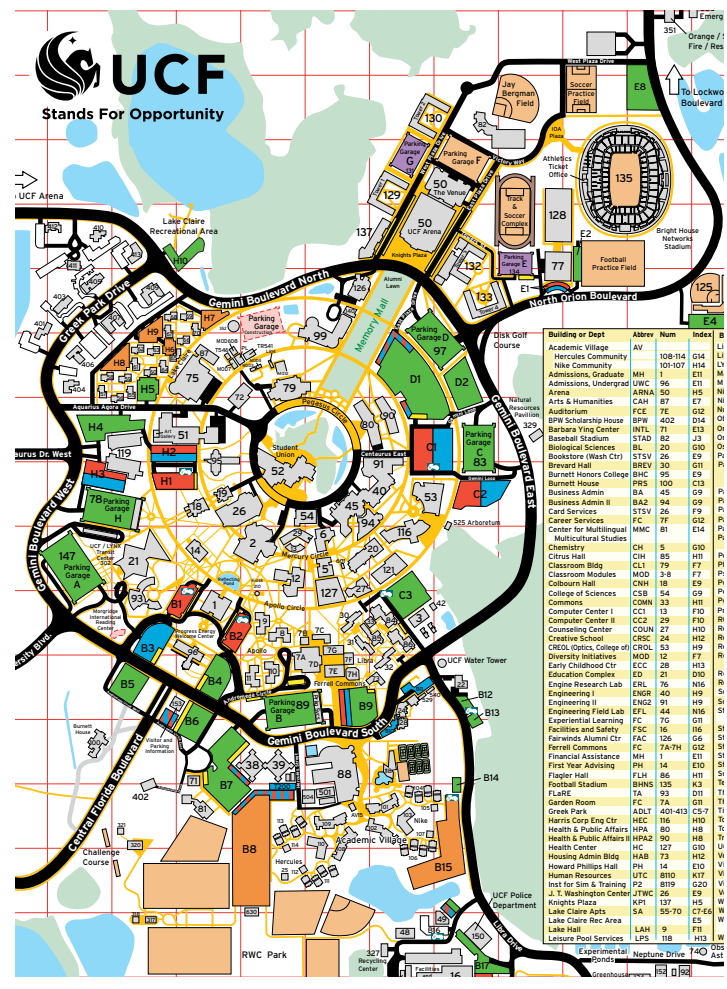

(a) UCF main campus

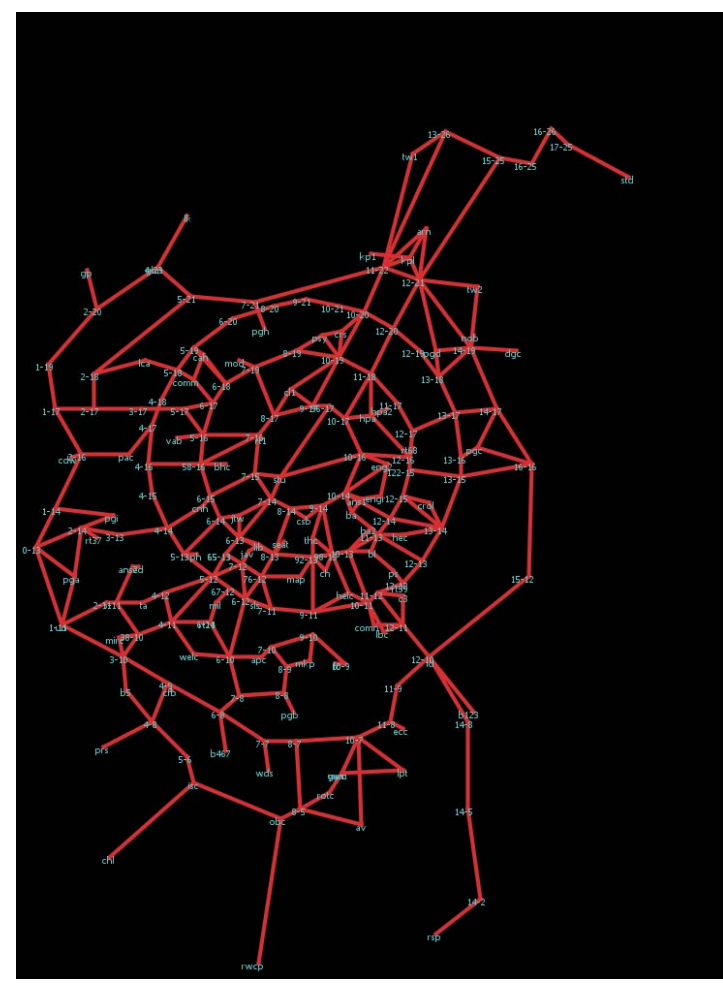

(b) Simulation graph

Fig. 1: The map used in the simulation along with the corresponding graph. Gray spots are buildings, black lines show the campus roads, and yellow lines indicate the walkways. Parking lots are marked in green (student), blue (staff), and red (faculty).

- ABM+survey: agent model parameters are randomly sampled from a set of continuous and discrete distributions that correspond to responses to survey questions. The parameters for these distributions are selected based on the best fit of the survey data.

Rather than directly mapping the survey data to simulated entities that match the exact preferences of one of the survey respondents, we attempt to learn a general model of the population by fitting a statistical distribution to the answers of every question. For those questions that were related to the time of visiting a location (e.g., campus arrival and departure times), a Gaussian distribution was used to create a continuous distribution of arrival and departure times for the population of agents. For those questions where the respondents provided frequencies (e.g., how often campus dining locations were visited), we evaluated the performance of several discrete distributions and selected to the Poisson distribution as offering the best fit for most of the questions. Figure 2 shows the fit of all of the 79 distributions used to initialize our ABM; the better fit distributions have negative log likelihoods falling closer to zero, shown in the figure as the shorter bars. Note that for our $\mathrm{ABM}$ we opted to use the same distribution model for all questions of a certain category regardless of the fit, rather than attempting to optimize the fit of the observed data by changing the form of the model. There was no discernible

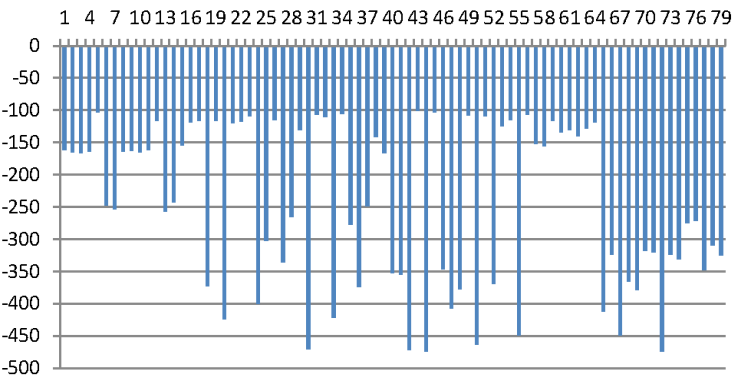

Fig. 2: The fit of the 79 distributions used to initialize the ABM (log likelihood vs. the question index). Better fit distributions have negative log likelihoods falling closer to zero, corresponding to a higher probability of the survey data being drawn from the distribution. The mean log likelihood over all the distributions is -244.1 , with a standard deviation of 123.5 .

interaction between the fit of the model by question category (e.g., dining patterns were better fitted by one model and parking lot preferences by another) so we used the same two distributions across all the questions.

After fitting the Poisson distribution on the qualitative data, 


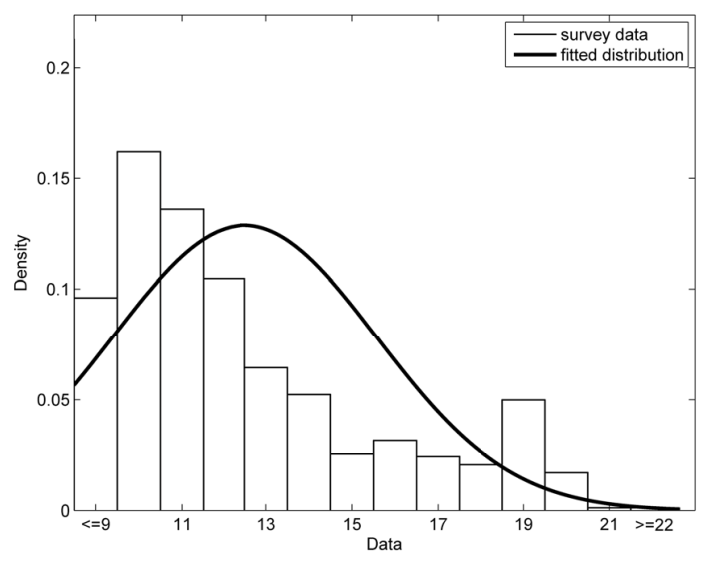

(a) Gaussian distribution for the entry time to the UCF main campus on Wednesdays. Columns correspond to the arrival time.

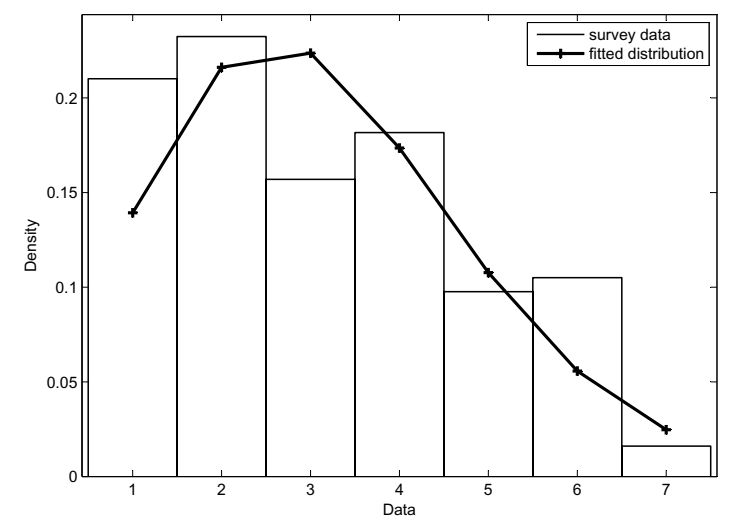

(b) Poisson distribution for the frequency of visiting dining locations in the Knights Plaza area of campus. Columns 1-7 are related to frequency of visits.

Fig. 3: Two fitted distributions used to initialize the agent populations in the proposed method (ABM+survey)

a mapping function is used to work with the values obtained. This function maps the qualitative frequencies to exact dates and times. Each term, from rarely to everyday, is treated separately. For instance, the term rarely is mapped to a random day in a 60 day period. Figure 3 shows two example distributions used to initialize the ABM for Wednesday campus entry times (Figure 3a) and the pattern of visiting dining locations in the Knights Plaza area of the campus (Figure $3 b$ ).

\section{Activity-oriented Microsimulation}

When the simulation commences, all the agents are initialized with parameters that remain constant over the lifetime of the agent and are used to create daily activity profiles. Our simulation is implemented in the Netlogo [7] environment. NetLogo (originally named StarLogo) is a high level platform, providing a simple yet powerful programming language, builtin graphical interfaces, and comprehensive documentation. It is particularly well suited for studying the evolution of complex systems over time [8].

In this environment, time is discrete and simulated by ticks where a tick is one unit of time. In our model, one tick represents one hour of activity in the real world. When the model starts, each agent runs within a loop. The loop continues until the simulation is stopped. Figure 4 shows the runtime process by which an agent activity profile is generated.

Based on the agent's parameters that are initialized at the beginning of the simulation, the agent activity profile generator determines what should an agent do and where should be at every time (tick). If sampling the agent's profile indicates that it should be on campus, then the function compares the current time with the possible activity times produced by the mapping function that maps frequencies from the agent's distribution model to specific times and dates. If a match is found, then the agent opts to travel to that location. Otherwise, the agent remains at its department as its default place. On the other hand, if the profile generator determines that the agent

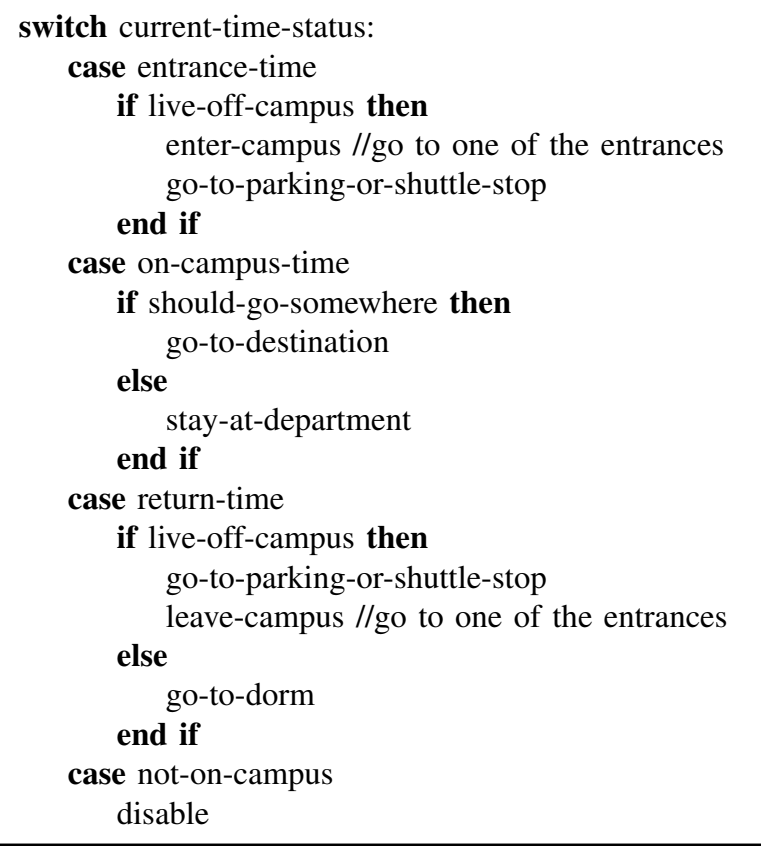

Fig. 4: Runtime generation of agent activity profiles

shouldn't be on campus, then the agent goes to (or remains in) the disabled state.

Various constraints are checked before an agent decides to go to a place. These constraints ensure the consistency of the whole model with the real world facts. The main consistency checks are summarized below:

- daily schedule: whenever an agent's model generates a date and time for visiting a location on campus, it 
checks the agent's arrival and departure times for that day. Campus activities that fall outside those boundaries are eliminated.

- activity overlap: whenever the agent's model generates trips that overlap in time, requiring the agent to be in multiple places at once, one of the overlapping tasks is shifted to a later time.

- campus constraints: known information about the operation hours of administrative offices, classroom buildings, and shuttle transportation is incorporated into the simulation. If the agent's model generates trips that violate the known operation hours, those trips are discarded.

A shortest path graph algorithm is used to choose the path that an agent should traverse between its start and end positions. To speed-up the model, an all pairs shortest path graph algorithm computes all of the shortest paths. A slightly modified version of Floyd-Warshall algorithm was used for this purpose. All path planning occurs at initialization; candidate paths are stored in a look-up table to be accessed later. The time complexity of Floyd-Warshall algorithm is $\theta\left(n^{3}\right)$. There are $n^{2}$ paths, and the length of each path is at most $n$, hence the space needed to store the paths (look-up table) is in the order of $O\left(n^{3}\right)$.

\section{Markov Chain Monte Carlo Simulation}

To compare the performance of our ABM model, we created a benchmark Markov Chain Monte Carlo simulation for making a limited set of forecasts based on the survey data. Markov Chain Monte Carlo describes a family of methods for performing Bayesian inferences using stochastic simulation. It has been used successfully in a wide variety of scientific [9] and engineering modeling applications [10]. MCMC allows us to draw samples from a distribution $\Pi(x)$ without having to know its normalization. With these samples, it is possible to compute any quantity of interest about the distribution of $x$, such as confidence regions, means, standard deviations, or covariance [11].

Rather than creating one large monolithic simulation of the entire urban system to explore a variety of scenarios, here MCMC is used to directly to forecast specific questions of interest, such as parking lot utilization. Our MCMC simulation uses the Metropolis-Hastings algorithm which randomly generates candidate points drawn from a proposal distribution around the existing points. It accepts candidate points with the following probability:

$$
\alpha\left(x_{e}, x_{c}\right)=\min \left(1, \frac{\Pi\left(x_{c}\right) q\left(x_{e} \mid x_{c}\right)}{\Pi\left(x_{e}\right) q\left(x_{c} \mid x_{e}\right)}\right)
$$

Here, $x_{e}$ is an existing point and $x_{c}$ is the candidate point. $\Pi(x)$ is the posterior distribution $\ddagger$ and $q(x)$ shows the proposal distribution. For more details about the MCMC method, the reader is referred to the following reference [2].

In this study, we have used the MCMC method as a benchmark to compare against the proposed agent-based model to

\footnotetext{
${ }^{\ddagger}$ More accurately stated, $\Pi(x)$ is a value proportional to the posterior distribution, the Bayes numerator.
}

\begin{tabular}{ll}
\hline Parameter & Value \\
\hline Agents & 47,000 \\
Days & 100 \\
Time Range & $07: 00-24: 00$ \\
\hline
\end{tabular}

TABLE I: The parameter settings of experiments

demonstrate the benefits of combining the higher fidelity ABM with the survey data. MCMC is used to estimate the number of cars entering the parking lots at different times of a day. One can envision this as a two dimensional diagram with the horizontal axis corresponding to the time of a day, and the vertical one showing the number of cars entering a specific parking lot. The survey data from the questions about the attendance pattern and frequency of parking lot usage are used to initialize the MCMC model. Observations for the Bayesian inference process are simply obtained based on the results of the survey data for a simulation period of 90 days. Imagine that based on the survey data a student respondent enters the campus everyday before 9 am, leaves at $5 \mathrm{pm}$, and reports his general usage of parking lot $\mathrm{A}$ as being at a frequency of once a week. In this case, the expectation is that the student would have occupied Lot A twelve times (90/7) during the simulation period so a corresponding number of samples tagged with the reported time range are produced and added to the input observation data.

The Metropolis-Hastings algorithm from the MCMC toolbox for Matlab [12] is used for the simulation. Our MCMC model assumes the prior is of the form of a Poisson distribution, the same as our ABM+Survey model. For the proposal distribution, a Gaussian is used. The MCMC attempts to find the most likely value of the the mean of the Poisson distribution $\left(\lambda\right.$ in $\left.\frac{\lambda^{x} e^{-\lambda}}{x !}\right)$.

\section{REsults}

To evaluate the performance of the agent-based model under different initialization conditions, we examined the transportation forecasts produced by the simulation, both through visualization and by comparing the predictions against a dataset collected by the UCF Parking Services office. The parameter values that are used in all of the experiments are listed in Table I.

One of the main applications of our microsimulation is analyzing pedestrian movement and car traffic on campus. Figure 5 shows the average visitation frequency for UCF campus locations (junctions, roads, and buildings) as predicted by our simulation. The size of the circles in $5 \mathrm{a}$ and $5 \mathrm{c}$, and the thickness of line in $5 \mathrm{~b}$ are proportional to the number of the agents who passed or visited these places.

Some obvious facts that can be easily verified by a domain expert are also observed in this set of results. For instance, as on most university campuses, the student union is the most frequently visited place since it is the venue for most events and many dining locations. The wide drivable boulevard that surrounds the campus dominates the road usage as it is the 


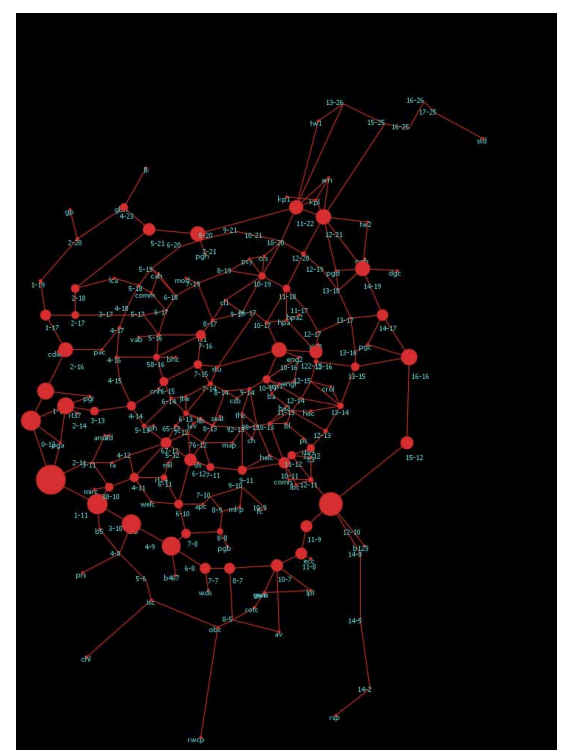

(a) Junctions

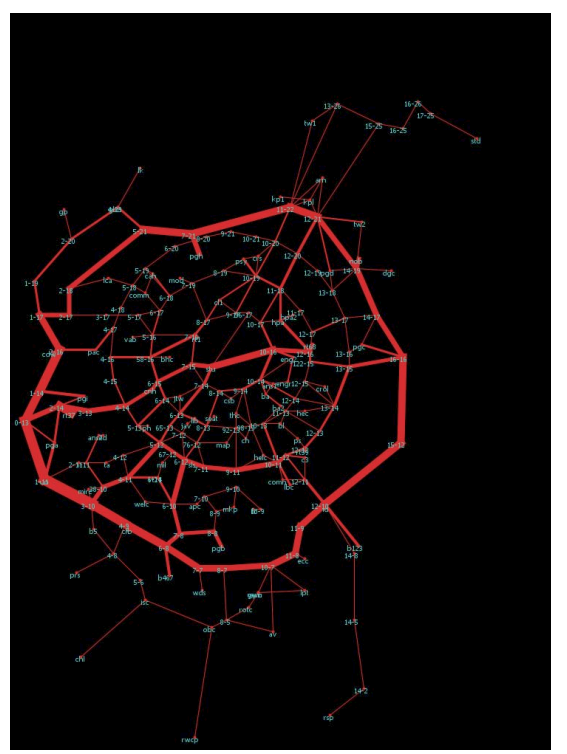

(b) Roads and walkways

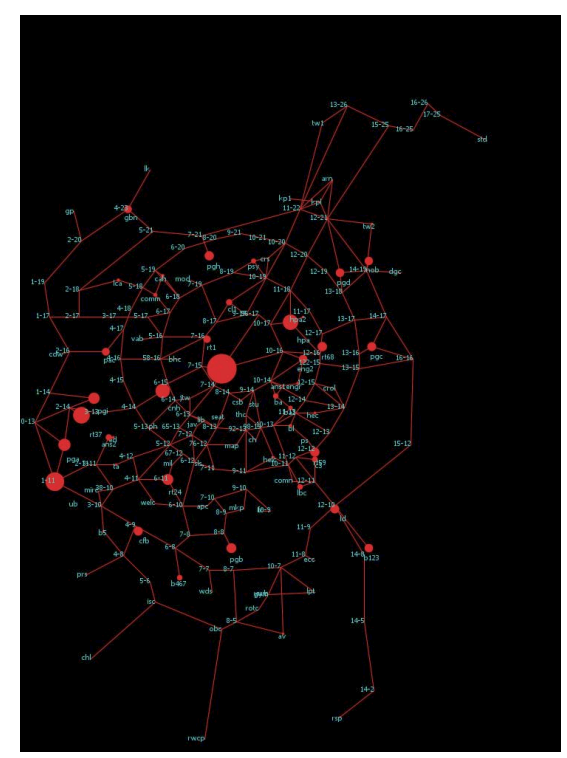

(c) Buildings

Fig. 5: Average traffic through different locations on the campus as predicted by the simulation. The simulation clearly shows several campus usage trends that are easily verified: 1) high usage of the circle road, the only drivable boulevard around the campus 2) high traffic at both main campus entrances (bottom left and right) 3) high student union usage (center), both of the building and incoming road 4) high traffic near the biggest parking lots on campus (two large circles in the bottom left).

only way that can be used by cars and shuttles to reach most points on campus.

A question of daily interest for most students is parking lot usage: which lots have vacancies and where can the best parking spots be found? UCF Parking Service performed a visual survey of lot usage in Fall 2011 and created a data set which we compared to our hourly microsimulation forecasts of student lot usage. Note that although we ask questions about parking preferences on the survey, the survey data alone is insufficient to directly reveal the hourly parking lot usage without the agent-based simulation or the MCMC.

Figures 6 and 7 show the microsimulation forecasts for the different student parking lots as predicted by: 1) ABM Only: the agent-based model initialized and simulated without survey data; 2) ABM+Survey: our proposed model in which the survey data is used to create the distributions for generating agent activity profiles; 3) MCMC: the Markov Chain Monte Carlo parking simulation 4) Empirical: the actual data provided by UCF Parking Services. The horizontal axis shows the names of the parking lots and the vertical the cars entering the lots during different time periods.

The results clearly show that the forecasts from the microsimulation are fairly close to the actual data collected for most of the lots. The one exception is the mismatch between the UCF Parking Service results and the forecast for Parking Lot A usage at 4pm. One likely explanation for the discrepancy is that an increase in student enrollment since the empirical data collection has caused a general increase in parking lot usage. The empirical data was collected in Fall

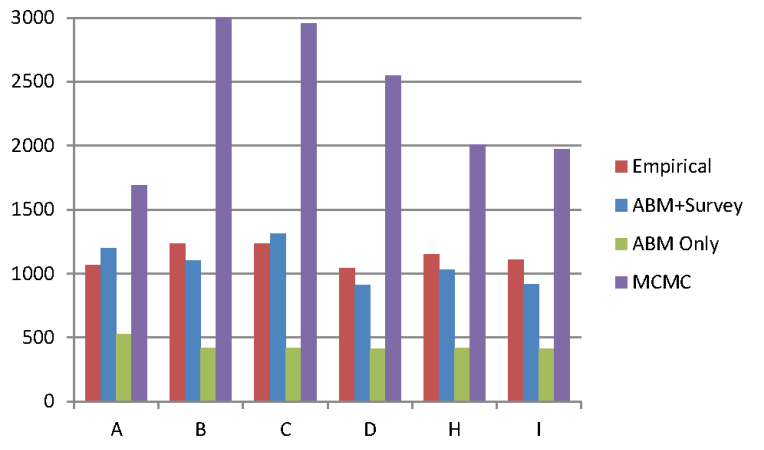

Fig. 6: The average number of cars entering the student parking lots at noon as predicted by the MCMC simulation (MCMC), the standard modeling method (ABM Only), our proposed method (ABM+Survey), and Empirical, the data from UCF Parking Services. Our proposed method is substantially more accurate at predicting parking lot utilization (as shown by the small difference between the red and blue bars) than the MCMC or the ABM without our initialization technique.

2011, while the survey and simulation data was gathered in the Spring 2012. Since Parking Lot A is a large, rarely fully occupied lot, it has a tendency to absorb overflow traffic. Another caveat is that the current version of our simulation does not model the movement patterns of the staff/faculty who are also allowed to park in student lots. An important 


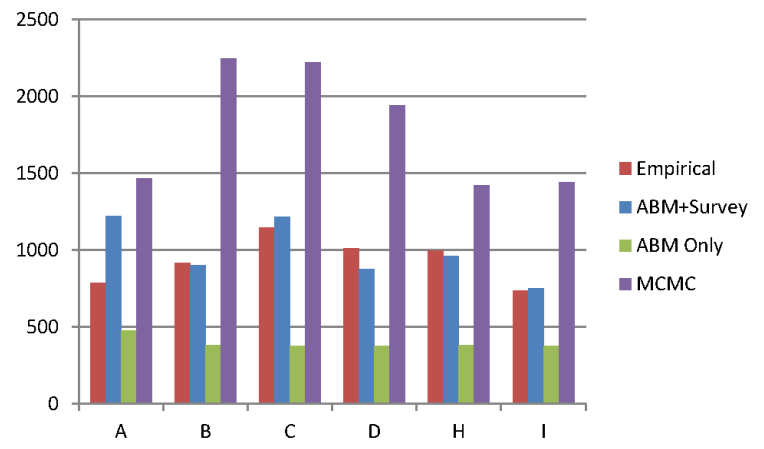

Fig. 7: The average number of cars entering the student parking lots at $4 \mathrm{PM}$ as predicted by the MCMC based simulation (MCMC), the standard modeling method (ABM Only), our proposed method (ABM+Survey), and Empirical, the data from UCF Parking Services. Our proposed method is the most successful at predicting the usage of most of the parking lots, shown by the small difference between the red and blue bars. The ABM alone underestimates the parking lot usage, whereas the MCMC predicts the trend of usage patterns but overestimates the number of cars.

thing to note is that the ABM model alone, initialized with a reasonable set of initial parameters, does not do a good job at forecasting parking lot usage, which is a relatively simple question. Without information about the times that the students are likely to be found on campus or their lot preferences, it predicts a fairly even spread of cars to lots. Also since it does not accurately model the time peaks in campus usage, it tends to predict an even spread of lot usage across all day time hours. The MCMC, which is initialized with the same survey data as our proposed method but lacks the detailed activity-based microsimulation, consistently overestimates the parking lot usage. However, it is more successful at expressing general peaks and dips in the occupancy that are missed by the ABM alone. None of the models use specific parking lot physical constraints (such as maximum capacity) nor time constraints (banned parking times) that would give them an unfair advantage in their calculations.

\section{RELATED WORK}

Agent-based models have been used to successfully model urban environments in a wide variety of applications including: 1) civil and environmental transportation analysis [13] 2) geographic information systems (GIS) for visualizing patterns and trends in spatial areas [14] and 3) archaeological studies of land site usage in ancient civilizations [15].

Although these systems do not necessarily have to accurately simulate physical interactions, incorporating spatial information and heterogeneity into agent-based models can improve our ability to draw conclusions about the behavior of complex systems in realistic environments, which may be different from conclusions drawn with artificial environments [16]. With the inclusion of GIS to represent a spatially, georeferenced environment, the impact of human behavior patterns can be linked to specific spatial locations and when used correctly can provide a powerful tool for policy makers and the public to understand the potential consequences of their decisions [17]. For instance, [18] presents an agent-based model for analyzing the influence of neighborhood design on daily trip patterns based on the detailed trip survey data from seven Traffic Analysis Zones (TAZs) in Ottawa, Canada. In [19], results obtained from a behavioral survey of driving behaviors were used to identify and fit a series of agent behavior parameters defining driver characteristics, knowledge and preferences; the authors also present a case study implementing a simple agent-based route choice decision model within a microscopic traffic simulation tool. However neither of those works present a systematic evaluation of different modeling techniques as was done here. In a different domain, modeling the diffusion of water-savings innovations, an ABM was calibrated using empirical data stemming from a questionnaire survey [20], showing that this technique can generalize across simulation domains.

In this paper, we describe the development of an activitybased microsimulation for modeling and forecasting transportation patterns on the UCF campus. For a complete survey of agent-based approaches to transportation and traffic management, the reader is referred to [21]. In many of these systems, each agent represents an individual person or vehicle, thus giving rise to the question of how to initialize the models to create behavior that is realistic in both the individual and aggregate sense. The four methods customarily employed are: 1) agents are randomly initialized using a reasonable range of parameters [13]; 2) recommendations from domain expert are used to guide parameter selection; 3) agents are designed to directly mimic actual members of the population [14]; 4) a hybrid combination of random initialization and expert guidance is employed at initialization [15]. In contrast, our simulation attempts to mirror the population using a series of fitted distributions rather than mimicking specific individuals within the population.

\section{CONCLUSION}

Although domain experts are an important part of the modeling process, in cases where it is possible to obtain data, it is desirable to reduce some of the subjectivity in parameter selection. Our initialization method of combining agent-based models with survey data allows us to streamline model creation, making the process more automatic. In this paper, we have presented the aspects of our campus modeling effort that apply to the widest possible range of urban microsimulations.

One simple improvement that we are planning to make in the future is to add faculty/staff into our simulation; this was not a priority initially since previous work has shown that faculty/staff activity profiles have a much lower entropy and are inherently easier to predict than student profiles [5]. Supplementing the simulation with additional information about semester class scheduling is likely to yield the largest forecasting improvement at the cost of making the simulation less 
applicable to other urban modeling problems. A large amount of class attendance and scheduling information is collected by the university and could be added to the simulation without requiring additional survey efforts.

\section{ACKNOWLEDGMENTS}

This research was supported by DARPA award N10AP20027 and AFOSR YIP FA9550-09-1-0525.

\section{REFERENCES}

[1] C. M. Macal and M. J. North, "Agent-based modeling and simulation: desktop ABMS," in Proceedings of the Conference on Winter Simulation. Piscataway, NJ, USA: IEEE Press, 2007, pp. 95-106.

[2] C. Andrieu, N. de Freitas, A. Doucet, and M. Jourdan, "An introduction to MCMC for machine learning," Machine Learning, vol. 50, pp. 5-43, 2003.

[3] J. Oakes, "Invited commentary: Rescuing Robinson Crusoe," American Journal of Epidemiology, vol. 8, no. 1, pp. 9-12, 2008.

[4] I. Benenson, P. Torrens, W. Europe, and J. Portugali, "Geosimulation: automata-based modeling of urban phenomena," Environment and Planning B: Planning and Design, vol. 31, no. 4, pp. 589-613, 2004.

[5] N. Eagle and A. Pentland, "Reality mining: sensing complex social systems," Pervasive and Ubiquitious Computing, vol. 10, pp. 255-368, 2006.

[6] J. Letchner, J. Krumm, and E. Horvitz, "Trip router with individualized preferences (TRIP): Incorporating personalization into route planning," in Proceedings of Conference on Innovative Applications of Artificial Intelligence, 2006.

[7] U. Wilensky, 1999, NetLogo. Evanston, IL: Center for Connected Learning and Computer-Based Modeling, Northwestern University. Retrieved from: http://ccl.northwestern.edu/netlogo/.

[8] R. Allan, "Survey of agent based modelling and simulation tools," Science and Technology Facilities Council, Tech. Rep. DL-TR-2010007, 2010.

[9] R. Liu, J. Tao, N. Shi, and X. He, "Bayesian analysis of the patterns of biological susceptibility via reversible jump MCMC sampling," Computational Statistics \& Data Analysis, vol. 55, no. 3, pp. 14981508, 2011.

[10] Y. Liu, Q. Wang, J. Liu, and T. Wark, "MCMC-based indoor localization with a smart phone and sparse wifi access points," in IEEE International Conference on Pervasive Computing and Communications Workshops (PERCOM Workshops), March 2012, pp. 247-252.

[11] W. Press, S. Teukolsky, W. Vetterling, and B. Flannery, Numerical Recipes 3rd Edition: The Art of Scientific Computing. Cambridge University Press, 2007.

[12] M. Laine, 2012, MCMC toolbox for Matlab, Finnish meteorological institute. Retrieved from: http://helios.fmi.fi/ lainema/mcmc/.

[13] X. Jin and L. Jie, "A study of multi-agent based models for urban intelligent transport systems," International Journal of Advancements in Computing Technology, vol. 4, no. 6, pp. 126-134, April 2012.

[14] R. Jordan, M. Birkin, and A. Evans, "Agent-based modelling of residential mobility, housing choice and regeneration," in Agent-Based Models of Geographical Systems. Springer Netherlands, 2012, pp. 511-524.

[15] T. A. Kohler, R. K. Bocinsky, D. Cockburn, S. A. Crabtree, M. D. Varien, K. E. Kolm, S. Smith, S. G. Ortman, and Z. Kobti, "Modelling prehispanic Pueblo societies in their ecosystems," Ecological Modelling, vol. 241, pp. 30-41, 2012.

[16] D. Brown, R. Riolo, D. Robinson, M. North, and W. Rand, "Spatial process and data models: Toward integration of agent-based models and gis," Journal of Geographical Systems, vol. 7, no. 1, pp. 25-47, 2005.

[17] H. Gimblett, Integrating geographic information systems and agentbased modeling techniques for simulating social and ecological processes. Oxford University Press, USA, 2002.

[18] X. Jin and R. White, "An agent-based model of the influence of neighbourhood design on daily trip patterns," Computers, Environment and Urban Systems, 2012.

[19] H. Dia, "An agent-based approach to modelling driver route choice behaviour under the influence of real-time information," Transportation Research Part C: Emerging Technologies, vol. 10, no. 5-6, pp. 331-349, 2002.
[20] N. Schwarz and A. Ernst, "Agent-based modeling of the diffusion of environmental innovations-An empirical approach," Technological forecasting and social change, vol. 76, no. 4, pp. 497-511, 2009.

[21] B. Chen and H. Cheng, "A review of the applications of agent technology in traffic and transportation systems," IEEE Transactions on Intelligent Transportation Systems, vol. 11, no. 2, pp. 485-497, June 2010. 